\title{
ITERATIVE SOURCE-CHANNEL DECODING USING SHORT BLOCK CODES
}

\author{
Thorsten Clevorn and Peter Vary \\ Institute of Communication Systems and Data Processing \\ RWTH Aachen University \\ \{clevorn,vary\}@ind.rwth-aachen.de
}

\author{
Marc Adrat \\ Dept. FKIE/KOM \\ FGAN, Wachtberg, Germany \\ adrat@fgan.de
}

\begin{abstract}
Iterative source-channel decoding (ISCD) exploits the residual redundancy of the source, e.g., codec parameters, for quality improvements. In contrast to the well-known convolutional coded ISCD systems, we propose in this paper an ISCD scheme which features a superior performance but is based solely on two short block codes. The block codes serve as highly redundant index assignment and rate-1 inner channel code respectively. The improved capabilities are confirmed by EXIT charts. Finally, the effects of imperfect knowledge of the receiver on the residual redundancy are analyzed. With the proposed flexible index assignment no feedback from the receiver to the transmitter is required and competitive results are obtained even for slightly erroneously estimated residual redundancy.
\end{abstract}

\section{INTRODUCTION}

With the discovery of Turbo codes channel coding close to the Shannon limit becomes possible with moderate computational complexity. In the past years the Turbo principle of exchanging extrinsic information between separate channel decoders has been adapted to other receiver components.

To exploit the residual redundancy in source coded parameters such as scale factors or predictor coefficients for speech, audio, and video signals in a Turbo process, iterative source-channel decoding (ISCD) $)^{1}$ has been presented in $[1,2]$. This residual redundancy occurs due to imperfect source encoding resulting from the delay constraints of, e.g., $20 \mathrm{~ms}$ in GSM. The a priori knowledge on the residual redundancy, e.g., non-uniform probability distribution or auto-correlation, is utilized for error concealment by a derivative of a soft decision source decoder (SDSD) [3], which exchanges extrinsic reliabilities with a channel decoder.

So far, ISCD schemes usually use an index assignment with no or low redundancy for the source codec parameters. The artificial redundancy for error correction is added afterwards by a convolutional channel coding with low rates $r_{\mathrm{C}}$, e.g., $r_{\mathrm{C}} \leq 1 / 2$. The convolutional code is applied to the complete frame of parameters. In this paper we propose a completely different approach. Instead of one convolutional code for the complete frame, we split the channel coding into two parts with short block codes and integrate the part which provides the major part of the artificial redundancy into the source (de)coder. This results in highly redundant index assignments [4] and rate- 1 explicit (inner) channel coding. Furthermore, due to the flexibility of the highly redundant index assignments this scheme can

\footnotetext{
The IND was supported by the Deutsche Forschungsgemeinschaft (DFG).

${ }^{1}$ The term iterative source-channel decoding is also used for the iterative evaluation of variable-length source codes and channel codes, where ISCD serves for a proper segmentation of the reconstructed bit stream after channel decoding into bit patterns of specific length.
}

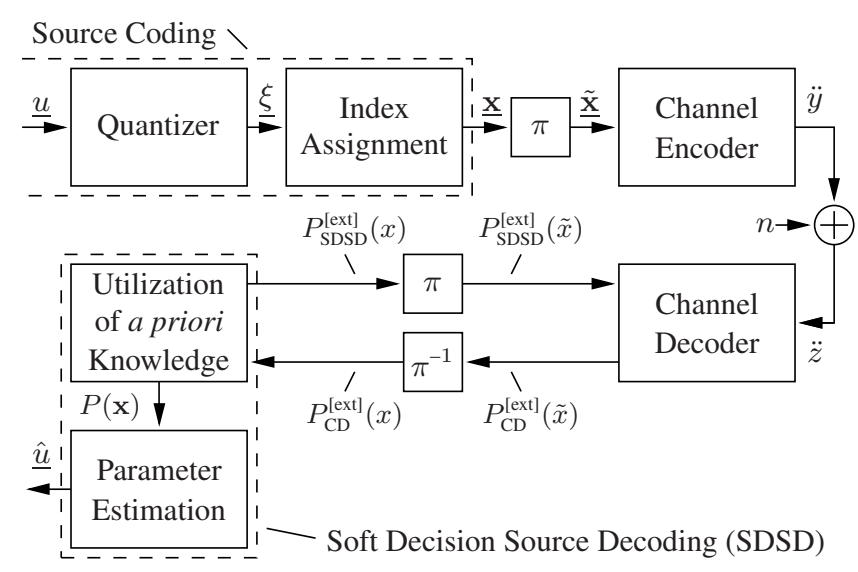

Fig. 1. Baseband model of iterative source-channel decoding

be very easily extended to a versatile multi-mode system, which allows a flexible trade-off between error robustness for bad channels and high quality for good channels.

After briefly reviewing conventional ISCD with convolutional coding in Section 2, we present the novel ISCD scheme, which is based on two very short block codes, in Section 3. In Section 4 we evaluate the capabilities of the proposed ISCD scheme in comparison to conventional ISCD by simulation results. Additionally, we analyze the effects of imperfectly estimated residual redundancy.

\section{CONVENTIONAL ISCD SYSTEM MODEL}

In Fig. 1 the baseband model of iterative source-channel decoding is depicted. At time instant $\tau$, a source encoder determines a frame $\underline{u}_{\tau}$ of $K_{S}$ source codec parameters $u_{\kappa, \tau}$ with $\kappa=1, \ldots K_{S}$ denoting the position in the frame. The single elements $u_{\kappa, \tau}$ of $\underline{u}_{\tau}$ are assumed to be statistically independent from each other. Each value $u_{\kappa, \tau}$ is individually mapped to a quantizer reproduction level $\bar{u}_{\kappa}^{\left(\xi_{\kappa}\right)}$ with index $\xi_{\kappa}=0, \ldots Q_{\kappa}-1$. The number of quantizer levels is usually assumed as $Q_{\kappa}=2^{M_{\kappa}}$. To each quantizer level $\bar{u}_{\kappa}^{\left(\xi_{\kappa}\right)}$ selected at time instant $\tau$ a unique bit pattern $\mathbf{x}_{\kappa, \tau}$ of $M_{\kappa}$ bits is assigned according to the index assignment $\Gamma, \mathbf{x}_{\kappa, \tau}=\Gamma\left(\xi_{\kappa}\right)$. For simplicity we assume $M_{\kappa}=M$ for all $\kappa$. The single bits of a bit pattern $\mathbf{x}_{\kappa, \tau}$ are indicated by $x_{\kappa, \tau}^{(m)}, m=1, \ldots M$. The frame of bit patterns is denoted by $\underline{x}_{\tau}$. The bit interleaver $\pi$ scrambles the incoming frame $\underline{\mathbf{x}}_{\tau}$ of data bits to $\underline{\tilde{\mathbf{x}}}_{\tau}$ in a deterministic manner. We restrict the interleaving to a single time frame with index $\tau$ and omit the time frame index $\tau$ in the following where appropriate.

For channel encoding $C$ of a frame $\underline{\tilde{x}}$ of bits $x$ we can use, e.g., a standard terminated recursive (non-) systematic convolutional code of constraint length $J+1$ and rate $r_{C}$. In general, any channel code can be used as long as the respective decoder is able to provide the 
required extrinsic reliabilities. For the termination of the convolutional code, $J$ tail bits are appended to $\underline{\underline{x}}$. The resulting codeword is denoted by $\mathbf{y}$ with bits $y$, which are mapped to bipolar bits $\ddot{y} \in\{ \pm 1\}$ for BPSK transmission with symbol energy $E_{s}=1$.

On the channel additive white Gaussian noise (AWGN) $n$ with a known power spectral density of $\sigma_{n}^{2}=N_{0} / 2$ is applied, i.e., $\ddot{z}=\ddot{y}+n$.

The received symbols $z_{k}$ are evaluated in a Turbo process, which exchanges extrinsic reliabilities between the channel decoder (CD) and the soft decision source decoder (SDSD). Such reliability information can either be evaluated in terms of probabilities $P(\cdot)$ or in log-likelihood ratios, or $L$-values, $L(\cdot)$.

The ISCD receiver is described in detail in $[1,2,5]$. For convolutional codes the equations for the computation of the extrinsic probabilities or their respective L-values are well known $[6,7]$. The SDSD determines extrinsic information mainly from the natural residual source redundancy which typically remains in the bit patterns $\mathbf{x}_{\kappa, \tau}$ after source encoding. Such residual redundancy appears on parameter-level, e.g., in terms of a non-uniform distribution $P\left(\mathbf{x}_{\kappa, \tau}\right)$, in terms of correlation, or in any other possible mutual dependency in time $\tau$. The latter terms of residual redundancy are usually approximated by a first order Markov chain, i.e., by the conditional probability distribution $P\left(\mathbf{x}_{\kappa, \tau} \mid \mathbf{x}_{\kappa, \tau-1}\right)$. These source statistics can usually be measured once in advance for a representative signal data base. The technique how to combine this a priori knowledge $P\left(\mathbf{x}_{\kappa, \tau} \mid \mathbf{x}_{\kappa, \tau-1}\right)$ on parameter-level with the soft-input values $P_{\mathrm{CD}}^{\text {[ext] }}(x)$ on bit-level is also well-known in the literature. The algorithm how to compute the extrinsic $P_{\mathrm{SDSD}}^{[\mathrm{ext}]}(x)$ has been detailed, e.g., in $[1,2,5]$.

\section{PROPOSED ISCD WITH BLOCK CODES}

In [8] it was analytically shown that (in case of a binary erasure channel) the inner component(s) of a serially concatenated Turbo scheme should be of rate $r=1$. ISCD with rate- 1 convolutional channel coding (called Turbo error concealment) has been studied in [9]. An index assignment with $M=\log _{2} Q$ bits was combined with a rate- 1 convolutional code. In this paper we use a different approach. The usual Trellis-based convolutional channel coding is removed. Instead, the artificial redundancy is applied by two short block codes, one with $r<1$ for a redundant index assignment and one with $r=1$ as (inner) channel code. However, Fig. 1 also depicts the model of the block coded ISCD scheme. Just the rates between $\xi, \mathbf{x}$, and $\ddot{y}$ change and the channel code is now a block code.

\subsection{Redundant index assignments}

Highly redundant index assignments for ISCD were proposed in [4]. A related concept for the non-iterative case has been presented in [10]. In comparison to a conventional ISCD scheme with channel coding of rate $r_{C}$, the number of bits assigned to each quantizer level $\bar{u}_{\kappa}^{\left(\xi_{\kappa}\right)}$ can be increased by a factor of $1 / r_{C}$, if we assume a constant gross bit rate. For example, if typical $r_{C}=1 / 2$ channel coding and $M=3$ bits per each of the $Q$ quantizer levels were used for conventional ISCD, with the proposed scheme bit patterns $\mathrm{x}$ of size $M^{\star}=M / r_{C}=6$ can be assigned to each quantizer level.

The obvious choice now would be to use $Q^{\star}=2^{M^{\star}}=64$ quantizer levels to reduce the quantization noise. However, the only constraint for $Q^{\star}$ is $Q^{\star} \leq 2^{M^{\star}}$. Thus, if, e.g., the quantization noise of $Q^{\star}=8$ quantization levels is tolerable (as in the conventional case with $M=3$ bits), we can apply a highly redundant index assignment with $M^{\star}=6$ bits for each level. The benefits of the latter approach with $Q^{\star}<2^{M^{\star}}$ over the obvious choice $Q^{\star}=2^{M^{\star}}$ in case of channel errors have been analyzed in detail in [4]. Such an index assignment with $Q^{\star}<2^{M^{\star}}$ can be considered as a (potentially non-linear) block code with the binary representation of the quantizer level index $\{\xi\}_{2}, \xi=0, \ldots Q^{\star}-1$, as input. In case of an index assignment by a linear block code with generator matrix $\mathbf{G}^{\Gamma}$ we get

$$
\mathbf{x}=\Gamma(\xi)=\left(\{\xi\}_{2}\right) \cdot \mathbf{G}^{\Gamma}
$$

For the exemplary $Q^{\star}=8$ and $M^{\star}=6$ we choose a simple $(6,3)$ block code with

$$
\mathbf{G}_{\mathrm{BC}(6,3)}^{\Gamma}=\left(\begin{array}{cccccc}
1 & 0 & 0 & 1 & 0 & 1 \\
0 & 1 & 0 & 1 & 1 & 0 \\
0 & 0 & 1 & 0 & 1 & 1
\end{array}\right) .
$$

This $\mathbf{G}^{\Gamma}$ provides a minimum Hamming distances of $d_{\min }=3$ between the valid bit patterns $\mathbf{x}$. With a minimum Hamming distance larger than 1, perfect a-priori information yields perfect extrinsic information. All bits except the one considered are known and the considered bit is then uniquely determined. Thus, the EXIT characteristic $[5,11]$ of the SDSD can reach the upper right corner, i.e., the point $(1,1)$, of the EXIT Chart [11]. Note that the computational complexity of the Trellis-based utilization of a priori knowledge in the SDSD does not increase significantly when increasing $M$ to $M^{\star}$ as the shape of the fully connected Trellis solely depends on the number of quantization levels (or states) $Q$ or $Q^{\star}$. Only the $M$ bits per Trellis edge are mapped to a pattern with $M^{\star}$ bits.

Furthermore, we do not need to restrict the number of quantization levels $Q^{\star}$ to powers of 2. Instead we can adjust $Q^{\star} \in \mathbb{N}$ gradually to achieve the tolerable quantization noise. This way we can build a flexible multi-mode system [4], which (if adapted) can provide always the best possible quality for a certain channel condition. The adaptation consists only of choosing the correct index assignment, e.g., from a lookup-table.

There exist several different methods for the optimization of the index assignment. The index assignments given in [4] are, e.g., optimized using EXIT charts [5] and represent a very non-linear block code. However, the proposed index assignments $\left(\mathrm{BC}_{M^{\star}}^{Q^{\star}}\right)$ based on linear block codes $\mathrm{G}^{\Gamma}$ can be easily derived in contrast to the EXIT optimized ones $\left(\mathrm{EO}_{M}^{Q}, \mathrm{EO}_{M^{\star}}^{Q^{\star}}\right)$, that require a complex optimization process, which additionally depends on the level of redundancy, e.g., the auto-correlation. In case $Q^{\star}$ is not a power of 2 , the $\mathbf{G}^{\Gamma}$ for the next higher power of 2 is used as basis. Then some code words have to be excluded. If the removal starts with the bit patterns $\mathbf{x}$ for the largest $\xi$, the remaining bit patterns can still be generated using the linear block code in (1).

\subsection{Rate-1 (inner) block code}

For the inner component of the Turbo process we propose the usage of short block codes, each applied to a bit pattern $\tilde{\mathbf{x}}^{\star}$ of size $I^{\star}$ out of $\underline{\tilde{x}}$. This can be also considered as a multi-dimensional mapping (MDM) for BPSK channel symbols [12] with the Turbo process then performing iterative demodulation. The extension to higher order modulation is presented in [13] and is denoted as Turbo DeCodulation. With MDMs an appropriate number of bits is mapped to a set (or vector) $\mathbf{y}$ of channel symbols rather than to a single channel symbol. For example, a bit pattern $\tilde{\mathbf{x}}^{\star}$ with $I^{\star}=4$ bits can be mapped to four BPSK symbols. Using the construction method for optimal MDMs given in [12] this can be seen as linear block precoding the bit pattern $\tilde{\mathbf{x}}^{\star}$ of size $I^{\star}$ to $\mathbf{y}^{\star}$ and regular BPSK mapping afterwards. With the criterion given in [12] we can derive the following 
rate-1 generator matrix for the short block code:

$$
\mathbf{G}_{\mathrm{OPT} 4}^{\mathrm{C}}=\left(\begin{array}{cccc}
0 & 1 & 1 & 1 \\
1 & 0 & 1 & 1 \\
1 & 1 & 0 & 1 \\
1 & 1 & 1 & 1
\end{array}\right)
$$

The optimal $\mathbf{G}_{\mathrm{OPT} I^{\star}}^{\mathrm{C}}$ for other values of $I^{\star}$ have a similar appearance, i.e., an almost all-one matrix with zeros only on the complete diagonal excluding the lower right entry. The encoded bit pattern $\mathbf{y}^{\star}$ can be obtained by

$$
\mathbf{y}^{\star}=\tilde{\mathbf{x}}^{\star} \cdot \mathbf{G}^{\mathrm{C}} .
$$

The complete multi-dimensional mapping can be easily stored in a lookup-table. At the receiver the extrinsic probabilities for the $i$-th bit $\tilde{x}_{k}^{\star(i)}$ of the $k$-th bit pattern $\tilde{\mathbf{x}}_{k}^{\star}$ are given by $[12,14]$

$P_{\mathrm{CD}}^{[\mathrm{ext}]}\left(\tilde{x}_{k}^{\star(i)}=b\right) \sim \sum_{\mathbf{y}_{k} \in \mathcal{Y}_{b}^{i}} P\left(\mathbf{y}_{k} \mid \mathbf{z}_{k}\right) \prod_{j \neq i} P_{\mathrm{SDSD}}^{[\mathrm{ext}]}\left(\tilde{x}_{k}^{\star(j)} \in \mathbf{y}_{k} \cdot\left(\mathbf{G}^{\mathrm{C}}\right)^{-1}\right)$,

where $\mathbf{z}_{k}$ is the received vector for $\mathbf{y}_{k}$. The set $\mathcal{Y}_{b}^{i}$ comprises all $\mathbf{y}_{k}$ for which the $i$-th bit of the respective $\tilde{\mathbf{x}}_{k}^{\star}=\mathbf{y}_{k} \cdot\left(\mathbf{G}^{\mathrm{C}}\right)^{-1}$ is $b$, with $b \in\{0,1\}$. The product is taken for the $P_{\mathrm{SDSD}}^{[\text {ext }]}\left(\tilde{x}_{k}^{\star(j)}\right)$ of this $\tilde{\mathbf{x}}_{k}^{\star}$.

In Table 1 we compare the computational complexity of the proposed scheme with short rate- 1 block codes of size $I^{\star}$ and convolutional codes with memory $J$. In the table the required additions and multiplications for the calculations of one $P_{\mathrm{CD}}^{[\text {ext }}(\tilde{x})$ in an iterations are listed, excluding the initializing operations, which are done only in the first iteration. Note, for large $I^{\star}$ the number of multiplications can be significantly reduced from $2^{I^{\star}} \cdot\left(I^{\star}-1\right)$ to $3 \cdot 2^{I^{\star}}-2^{I^{\star}} / I^{\star}$, by first computing the product in (5) for all $j$ and afterwards exclude the respectively considered value $i$ by division (or multiplication with the one-time per symbol vector calculated inverse $\left.1 / P_{\mathrm{CD}}^{[\mathrm{ext}]}(\tilde{x})\right)$. It can be seen in Table 1 that, e.g., channel decoding for the proposed block coded scheme with $I^{\star}=4$ is slightly less complex than for a memory $J=3$ convolutional code. Furthermore, the short block codes allow easy parallelization because each block coded segment can be decoded independently.

\section{SIMULATION RESULTS}

\subsection{Comparison of proposed and conventional ISCD}

The capabilities of the proposed block coded iterative sourcechannel decoding (BC-ISCD) scheme compared to the conventional convolutional coded scheme (CC-ISCD) shall be demonstrated by simulation. The parameter signal-to-noise ratio (SNR) between the originally generated parameters $u_{\kappa}$ and the reconstructed estimates $\hat{u}_{\kappa}$ is used for quality evaluation. Instead of using any specific speech, audio, or video encoder, we model $K_{S}=250$ statistically independent source codec parameters $u$ by $K_{S}$ independent Gauss processes with $\sigma_{u}^{2}=1$. Thus, for the time being, the residual redundancy of the source consists only of a non-uniform parameter distribution but does not comprise correlation.

Each parameter $u_{\kappa}$ is scalarly quantized by a $Q=Q^{\star}=8$ level Lloyd-Max quantizer using $M=3$ or $M^{\star}=6$ bits/parameter. As

Table 1. Required operations per $P_{\mathrm{CD}}^{[\mathrm{ext}]}(\tilde{x})$ per iteration.

\begin{tabular}{|c||c|c||c|c|}
\hline \multicolumn{1}{|c||}{} & \multicolumn{2}{c||}{ proposed block code } & \multicolumn{2}{c|}{ convolutional code } \\
operation & in general & $I^{\star}=4$ & in general & $J=3$ \\
\hline+ & $2^{I^{\star}}$ & 16 & $3 \cdot 2^{J}-2$ & 22 \\
\hline$*$ & $2^{I^{\star}} \cdot\left(I^{\star}-1\right)$ & 48 & $8 \cdot 2^{J}$ & 64 \\
\hline
\end{tabular}

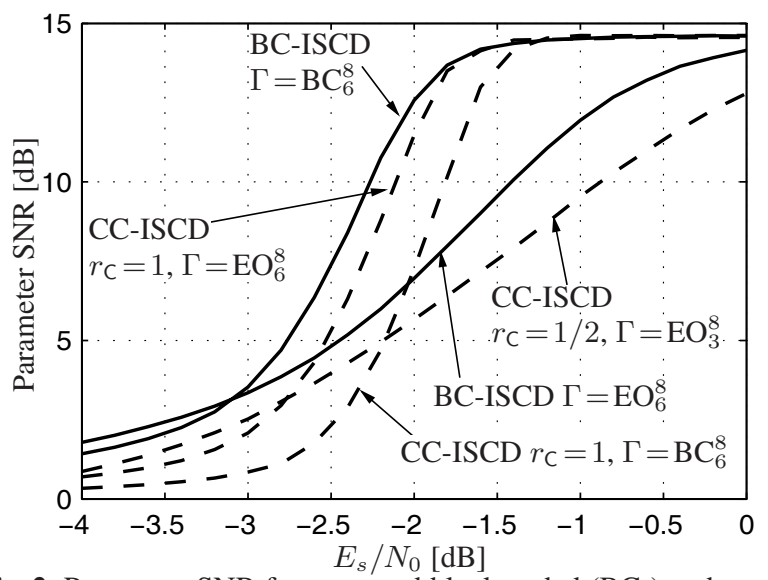

Fig. 2. Parameter SNR for proposed block coded (BC-) and conventional convolutional coded (CC-) ISCD.

index assignment $\Gamma: \xi \mapsto \mathbf{x}$ for the iterative schemes serves either the EXIT optimized $\left(\mathrm{EO}_{M}^{Q}, \mathrm{EO}_{M^{\star}}^{Q^{\star}}\right)$ index assignments presented in $[4,5]$ or the proposed block code based index assignment $\mathrm{BC}_{M^{*}}^{Q^{\star}}$. Note that the EXIT optimized index assignments always need to be optimized for the present auto-correlation $\rho$, i.e., $\rho=0$ in this case.

For channel coding $\mathrm{C}: x \mapsto y$ we assume as reference $r_{\mathrm{C}}=$ $1 / 2$, resulting in a random bit interleaver $\pi$ of size $M K_{S} / r_{\mathrm{C}}=$ 1500. Note, for a Turbo process the frame size of 1500 bits is relatively short. As block code for BC-ISCD we use $\mathbf{G}_{\text {OPT4 }}^{\mathrm{C}}$ and for CC-ISCD we consider memory $J=3$ recursive non-systematic convolutional codes with the generator polynomials $\mathbf{G}_{r_{\mathrm{C}}=1 / 2}^{\mathrm{C}}=\left(\frac{1+D+D^{3}}{1+D+D^{2}+D^{3}}, \frac{1+D^{2}+D^{3}}{1+D+D^{2}+D^{3}}\right)$ for $r_{\mathrm{C}}=1 / 2$ and $\mathbf{G}_{r_{C}=1}^{\mathrm{C}}=\left(\frac{1}{1+D+D^{2}+D^{3}}\right)$ for $r_{\mathrm{C}}=1$. Thus, the computational complexity is slightly less for BC-ISCD than for CC-ISCD (cmp. Tab. 1).

In Fig. 2 the parameter SNR after 25 iterations for BC-ISCD (solid curves) and CC-ISCD (dashed curves) is depicted. Fig. 3 contains the respective EXIT charts [11] for $E_{s} / N_{0}=-2.5 \mathrm{~dB}$ to demonstrate the convergence behavior. As visible, the proposed $\mathrm{BC}$ ISCD with $\mathrm{BC}_{6}^{8}$ exhibits the best performance. It preserves the maximum parameter SNR longer than the other schemes and possesses relatively smooth degradation towards low $E_{s} / N_{0}$, too. The corresponding EXIT chart in Fig. 3a illustrates that the slightly S-shaped EXIT characteristic of $\mathrm{BC}_{6}^{8}$ matches very well to the one of $\mathbf{G}_{\mathrm{OPT} 4}^{\mathrm{C}}$. In contrast, the almost linear EXIT characteristic of $\mathrm{EO}_{6}^{8}$, which also reaches the point $(1,1)$, yields a stopping intersection at low mutual information $\mathcal{I}$ (see Fig. 3b). Due to the relatively linear EXIT characteristic of $\mathbf{G}_{r_{C}=1}^{C^{C}}$, the situation is vice versa for CC-ISCD with $r_{\mathrm{C}}=1$. Here, the EXIT characteristic of $\mathrm{EO}_{6}^{8}$ (see Fig. 3d) matches better than the one of $\mathrm{BC}_{6}^{8}$ (see Fig. 3c). Consequently, CC-ISCD with $\mathrm{EO}_{6}^{8}$ surpasses CC-ISCD with $\mathrm{BC}_{6}^{8}$ as visible in Fig. 2. At high parameter SNRs both $r_{\mathrm{C}}=1$ schemes, BC-ISCD as well as CC-ISCD, significantly outperform classic CC-ISCD with $r_{\mathrm{C}}=1 / 2$. $\mathrm{BC}$-ISCD is superior in the whole $E_{s} / N_{0}$-range.

\subsection{Effects of imperfect knowledge on the auto-correlation}

The source codec parameters at the transmitter shall now exhibit an additional residual redundancy in terms of an auto-correlation $\rho_{\mathrm{t}}$. For the simulation, the parameters $u_{\kappa}$ are modeled now by independent first order Gauss-Markov processes with the respective $\rho_{\mathrm{t}}$. As exemplary $\rho_{\mathrm{t}}$ we chose $\rho_{\mathrm{t}}=0.4$ and $\rho_{\mathrm{t}}=0.7$ in addition to the previous case without correlation, i.e., $\rho_{\mathrm{t}}=0.0$. Such or even higher values can be found in real-world applications. However, to success- 

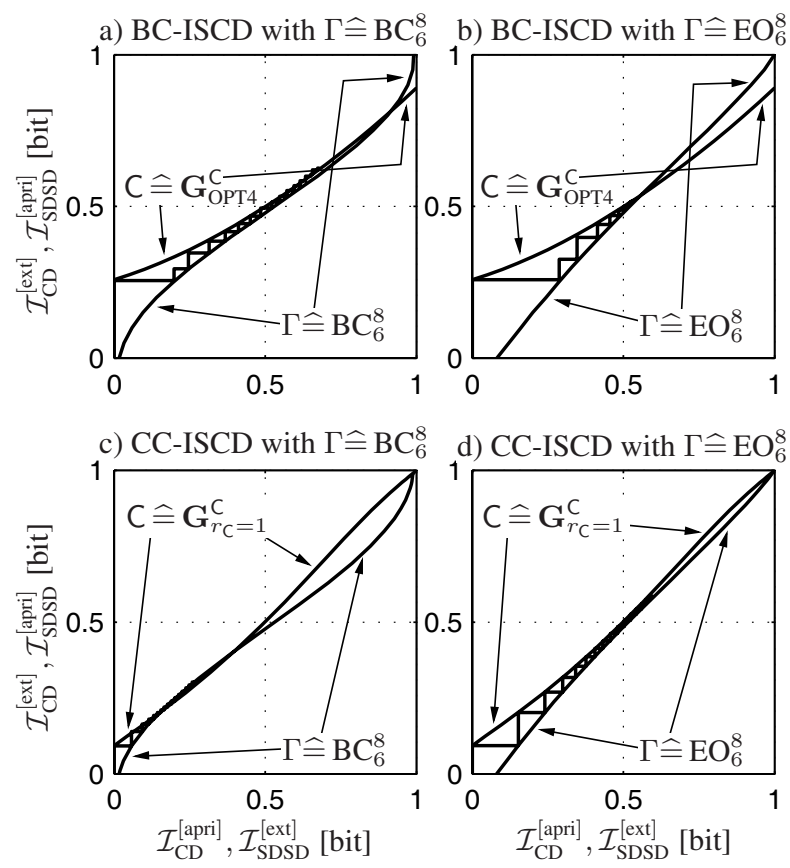

Fig. 3. EXIT Charts for BC-ISCD and $r_{\mathrm{C}}=1$ CC-ISCD in Fig. 2 at $E_{s} / N_{0}=-2.5 \mathrm{~dB}$.

fully exploit the additional residual redundancy, the receiver needs to know how much residual redundancy the parameters contain. In Fig. 4 the dashed lines represent simulation results for the case when the assumed auto-correlation at the receiver $\rho_{\mathrm{r}}$ does not match the actual auto-correlation at the transmitter $\rho_{\mathrm{t}}$, i.e., $\rho_{\mathrm{t}} \neq \rho_{\mathrm{r}}$. The solid lines indicate the results for a correct $\rho_{\mathrm{r}}$, i.e., $\rho_{\mathrm{t}}=\rho_{\mathrm{r}}$. All curves are labeled by $\left(\rho_{\mathrm{t}}, \rho_{\mathrm{r}}\right)$. As visible, for a correct $\rho_{\mathrm{r}}=\rho_{\mathrm{t}}$ the performance improves significantly. Up to $\Delta_{E_{s} / N_{0}} \approx 0.8 \mathrm{~dB}$ can be achieved for $\rho_{\mathrm{r}}=\rho_{\mathrm{t}}=0.7$.

In case the auto-correlation is slightly overestimated at the receiver, $\rho_{\mathrm{t}}<\rho_{\mathrm{r}}$, only a small loss of $\Delta_{E_{\mathrm{s}} / N_{0}} \lesssim 0.2 \mathrm{~dB}$ can be observed, e.g., for $\left(\rho_{\mathrm{r}}=0.7, \rho_{\mathrm{t}}=0.4\right)$ and $(0.4,0.0)$. However, for a significant overestimation $\rho_{\mathrm{t}} \ll \rho_{\mathrm{r}}$, e.g., $(0.0,0.7)$, a severe impairment is visible.

When $\rho_{\mathrm{r}}$ is underestimated, e.g., $(0.7,0.4)$, the performance degrades slightly. The redundancy by auto-correlation is not fully ex-

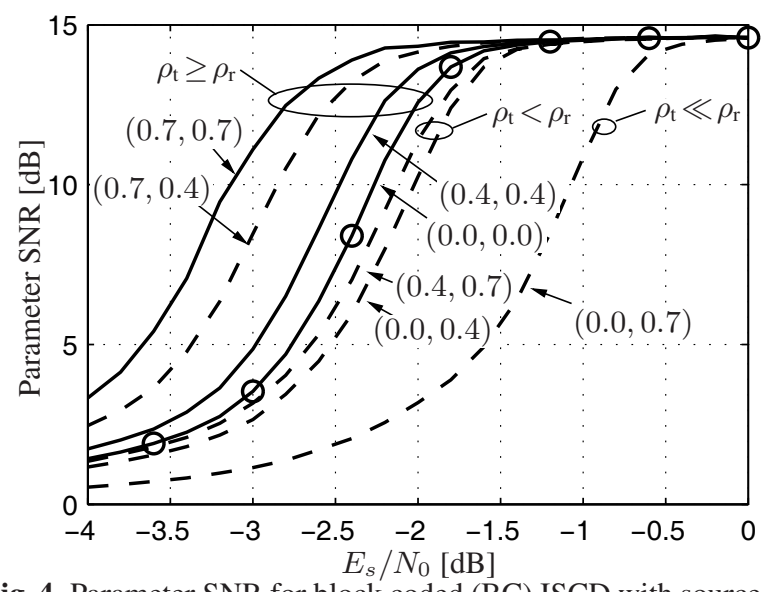

Fig. 4. Parameter SNR for block coded (BC) ISCD with source autocorrelation $\rho_{\mathrm{t}}$ at the transmitter and assumed auto-correlation $\rho_{\mathrm{r}}$ at the receiver (25 iterations). Curves labeled by $\left(\rho_{\mathrm{t}}, \rho_{\mathrm{r}}\right)$. ploited. But the scheme still surpasses the results for a lower but correct $\rho_{\mathrm{r}},(0.4,0.4)$ and $(0.0,0.0)$. All curves for $\rho_{\mathrm{t}}>0.0$ but $\rho_{\mathrm{r}}=0.0$ would coincide with $(0.0,0.0)$. Despite their auto-correlation $\rho_{\mathrm{t}}>0.0$ the parameters still exhibit the non-uniform Gaussian distribution expected by the receiver for any $\rho_{\mathrm{r}}$. Thus, for $\rho_{\mathrm{t}} \geq \rho_{\mathrm{r}}$ the performance can not deteriorate below the reference for $\rho_{\mathrm{r}}$.

In an application the receiver could initially assume $\rho_{\mathrm{r}}=0.0$ and in the following adjust to the auto-correlation it measures in its own processed signals. As the proposed block code based index assignments BC require no adaptation to the auto-correlation $\rho_{\mathrm{t}}$ (in contrast to the EXIT optimized index assignment EO), no feedback channel to the transmitter is necessary. To avoid the impairments occurring for $\rho_{\mathrm{t}} \ll \rho_{\mathrm{r}}$, a varying $\rho_{\mathrm{t}}$ should be slightly underestimated.

\section{CONCLUSION}

In this paper we presented a novel ISCD scheme, which is based on two very short linear block codes rather than on convolutional codes. One block code provides a highly redundant index assignment and the other acts as rate- 1 inner channel code, resembling a multi-dimensional mapping. The simulation results indicate that with the proposed flexible index assignment previously known convolutional coded ISCD systems with a similar computational complexity are outperformed by the presented block code based ISCD. These results are confirmed by an EXIT chart analysis. Furthermore, the effects of imperfect knowledge at the receiver on the residual redundancy are studied, revealing, e.g., a severe degradation for a significantly overestimated auto-correlation.

\section{REFERENCES}

[1] M. Adrat, P. Vary, and J. Spittka, "Iterative Source-Channel Decoder Using Extrinsic Information from Softbit-Source Decoding," IEEE ICASSP, Salt Lake City, Utah, May 2001.

[2] N. Görtz, "On the Iterative Approximation of Optimal Joint SourceChannel Decoding," IEEE J. Select. Areas Commun., Sept. 2001.

[3] T. Fingscheidt and P. Vary, "Softbit Speech Decoding: A New Approach to Error Concealment," IEEE Trans. Speech Audio Proc., Mar. 2001.

[4] M. Adrat, P. Vary, and T. Clevorn, "Optimized Bit Rate Allocation for Iterative Source-Channel Decoding and its Extension towards MultiMode Transmission," 14th IST Mob. \& Wirel. Comm. Summit, Dresden, Germany, June 2005.

[5] M. Adrat and P. Vary, "Iterative Source-Channel Decoding: Improved System Design Using EXIT Charts," EURASIP J. on Appl. Signal Proc. (Special Issue: Turbo Processing), May 2005.

[6] L. R. Bahl, J. Cocke, F. Jelinek, and J. Raviv, "Optimal Decoding of Linear Codes for Minimizing Symbol Error Rate," IEEE Trans. Inform. Theory, Mar. 1974.

[7] S. Benedetto, G. Montorsi, D. Divsalar, and F. Pollara, "Soft-Input Soft-Output Modules for the Construction and Distributed Iterative Decoding of Code Networks," Europ. Trans. Telecommun. (ETT), Mar. 1998.

[8] A. Ashikhmin, G. Kramer, and S. ten Brink, "Extrinsic information transfer functions: model and erasure channel properties," IEEE Trans. Inform. Theory, Nov. 2004.

[9] M. Adrat and P. Vary, "Iterative Source-Channel Decoding with Code Rates near r=1," IEEE ICC, Paris, France, June 2004.

[10] S. Heinen and P. Vary, "Source-Optimized Channel Coding for Digital Transmission Channels," IEEE Trans. Comm., May 2005.

[11] S. ten Brink, "Convergence Behavior of Iteratively Decoded Parallel Concatenated Codes," IEEE Trans. Comm., Oct. 2001.

[12] F. Simoens, H. Wymeersch, H. Bruneel, and M. Moeneclaey, "Multidimensional Mapping for Bit-Interleaved Coded Modulation with BPSK/QPSK Signaling," IEEE Comm. Lett., May 2005.

[13] T. Clevorn, M. Adrat, and P. Vary, "Turbo DeCodulation using Highly Redundant Index Assignments and Multi-Dimensional Mappings," 4th Intern. Symp. on Turbo Codes \& Related Topics, Munich, Germany, Apr. 2006.

[14] N. Gresset, J. J. Boutros, and L. Brunel, "Multidimensional Mappings for Iteratively Decoded BICM on Multiple-Antenna Channels," IEEE Trans. Inform. Theory, Sept. 2005. 